\title{
Substituição total do milho pela polpa cítrica no concentrado de bezerros leiteiros
}

\author{
Coimbra, E.P.; Azevedo, R.A.; Reis, R.B.; Saturnino, H.M. e Coelho, S.G.@
}

Escola de Veterinária. Universidade Federal de Minas Gerais. Minas Gerais. Brasil.

\section{PALAVRAS-CHAVE ADICIONAIS}

Ácido graxo volátil.

Coproduto.

Glicose.

Rúmen.

\section{RESUMO}

Objetivou-se avaliar os efeitos da substituição do milho pela polpa cítrica no concentrado sobre o desempenho e os parâmetros ruminais de bezerros até 60 dias de idade. Foram utilizados bezerros Holandês ( $n=36$ ), sendo 28 para as análises de consumo e desempenho e 8 para estudo dos parâmetros da fermentação ruminal e perfil da glicose sanguínea. Ao final, 4 animais de cada tratamento foram eutanasiados para análises do epitélio ruminal. O consumo de concentrado diferiu entre os tratamentos da $5^{a}$ à $9^{a}$ semana de idade, sendo maior para o grupo alimentado com concentrado com milho. O ganho de peso e a concentração sanguínea de glicose foram semelhantes entre os tratamentos. A concentração molar de acetato, propionato, butírico, total de AGV (ácidos graxos voláteis) e os valores de $\mathrm{pH}$ ruminal não diferiram entre os grupos. O maior percentual de propionato e o butirato foram observados respectivamente para os bezerros com dieta à base de milho e à base de polpa cítrica. Pequenas alterações anato-patológicas foram encontradas no epitélio ruminal dos bezerros alimentados com polpa cítrica no concentrado. Os resultados sugerem que a polpa cítrica é um coproduto com potencial de ser utilizada em substituição ao milho no concentrado de bezerros até 60 dias de idade.

\section{Replacement of corn by citrus pulp in the concentrate of dairy calves}

\section{SUMMARY}

\section{ADDITIONAL KEYWORDS}

Byproduct.

Glucose.

Rumen.

Volatile fatty acid.

INFORMATION

Cronología del artículo.

Recibido/Received: 29.08.2016

Aceptado/Accepted: 21.02.2017

On-line: 15.07 .2017

Correspondencia a los autores/Contact e-mail:

omvelezt@unal.edu.co

\section{INTRODUÇÃO}

A elevação e a oscilação nos preços dos componentes dos concentrados pode ser fator limitante na criação de bezerros. Desta forma, a busca de alternativas viáveis para a substituição de alguns ingredientes por alimentos alternativos de menor custo e que sejam capazes de manter a produção dos rebanhos é de interesse de técnicos e produtores, visando redução dos custos e consequentemente a viabilização da criação desses animais (Pimentel et al., 2012).
Uma alternativa é a substituição dos alimentos convencionais por coprodutos na formulação de dietas para animais em produção (Azevedo et al., 2014), os quais podem consistir em fontes interessantes de proteína, energia e fibra, além da sua utilização ser fomentada em razão de questões ambientais e econômicas (Azevedo et al., 2012). Os coprodutos são alimentos alternativos para a alimentação de ruminantes, os quais apresentam capacidade de utilizar resíduos vegetais para suprir seus requisitos nutricionais, além de substituírem, de forma parcial ou total, os alimentos padrões 
das dietas, como o milho e o farelo de soja (Pires et al., 2005; Silva et al., 2005).

A polpa cítrica é um coproduto fibroso da indústria de suco de laranja e tem sido utilizada na alimentação de ruminantes em substituição parcial ou total ao milho (Santos et al., 2014; Peixoto et al., 2015; Javed et al., 20016), sendo um alimento rico em sacarose, pectina e fibra de boa digestibilidade, o que possibilita a sua utilização na alimentação de vacas leiteiras (Santos et al., 2001; Nussio et al., 2002; Costa et al., 2014, Santos et al., 2014), novilhas (Mendes Neto et al., 2007) e de bezerros (Schalch et al., 2001). Porém, a composição proteica desse coproduto, em geral, é desequilibrada, sendo deficiente em aminoácidos, principalmente a metionina, e em fósforo, além de possuir altos conteúdos de cinzas e de cálcio, sendo este último muito variável, o que dificulta o balanceamento das dietas. Sendo assim, o fornecimento de polpa cítrica para animais jovens é questionável e possui diferentes indicações quando a quantidade de inclusão nas dietas desses animais. Harris Jr. and Staples (1989) recomendaram até 10\% de inclusão de polpa cítrica na dieta para bezerros, já Schalch et al. (2001) relatou que a polpa cítrica pode substituir até $100 \%$ do milho, em concentrados peletizados com adição de $5 \%$ de leite em pó desnatado, para bezerros durante a fase de aleitamento.

Objetivou-se com esse estudo avaliar os efeitos da substituição do milho pela polpa cítrica no concentrado e os seus efeitos sob o consumo de concentrado, desempenho, parâmetros ruminais e o perfil sanguíneo de glicose de bezerros leiteiros até 60 dias de idade.

\section{MATERIAL E MÉTODOS}

Todos os animais foram tratados como descrito no Guia para o Cuidado e Uso de Animais na Pesquisa Agropecuária e Ensino (FASS, 2010). O experimento foi realizado em uma fazenda comercial, Sete Lagoas, MG, Brasil.

Foram utilizados bezerros da raça Holandês ( $\mathrm{n}=$ 36), sendo 22 machos e 14 fêmeas, com peso corporal ao nascimento $(\mathrm{PCN})$ de 42,2 $\pm 4,31$ e 38,9 $\pm 3,97 \mathrm{~kg}$ para machos e fêmeas, respectivamente. Logo após o nascimento os bezerros foram separados de suas mães, sendo realizada a imersão do umbigo em solução de iodo (7\%), fornecimento de $3 \mathrm{~L}$ de colostro até $6 \mathrm{~h}$ após o nascimento, pesagem e identificação.

Entre o segundo e terceiro dia de idade os animais foram alimentados com $4 \mathrm{~L} / \mathrm{d}$ de leite de transição, em duas refeições iguais (08 e 15 h). No quarto dia de idade os animais foram aleatoriamente distribuídos $(n=18$ por grupo) em dois tratamentos, procurando manter o balanceamento quanto ao PCN e sexo. Os tratamentos consistiram de concentrado a base de milho ou a base de polpa cítrica como fonte energética (tabela I). Sabugo de milho foi incluído no concentrado a base de milho como forma de elevar os teores de FDA da dieta (tabela I). Entre o quarto e sétimo dia de vida todos os bezerros receberam $4 \mathrm{~L} / \mathrm{d}$ de leite integral, em duas refeições (08 e 15 h). A partir do oitavo dia de idade os bezerros passaram a receber $4 \mathrm{~L} / \mathrm{d}$ em uma única refeição (8 h). Aos 30 dias de idade os animais foram desaleitados de forma abrupta. Água e os diferentes tipos de concentrado foram fornecidos a vontade. Todos os animais foram mantidos em bezerreiro com abrigos individuais, sobre cobertura vegetal de Cynodon sp. até os 59 dias de idade.

O consumo de concentrado (g MS/dia) foi obtido diariamente por diferença entre a quantidade fornecida e as sobras. A partir de quatro dias de idade, semanalmente, antes do fornecimento das dietas, os animais foram pesados em balança (Coima, Dracena, São Paulo, Brasil).

Dos 11 bezerros machos de cada grupo experimental, quatro por grupo foram aleatoriamente selecionados para coletas de conteúdo ruminal. As coletas ocorreram aos 14, 24, 34, 44 e 54 dias de idade (ás 14 h). O material foi coletado por sonda oral, para análise de $\mathrm{pH}$ e AGV. No mesmo período foram realizadas coletas de sangue para análise de glicose plasmática (ás 7:30 h).

Logo após a coleta foi mensurado o pH do conteúdo ruminal em potenciômetro portátil (DM-2-Digimed, Campo Grande, Brasil). Para a análise de AGV, as amostras de fluido ruminal foram filtradas em gases, sendo adicionado ácido metafosfórico a 20\%, na proporção de quatro para um, sendo as amostras mantidas em freezer $\left(-20^{\circ}\right)$ até o momento da análise. No momento da análise as amostras foram descongeladas à temperatura ambiente, centrifugadas a 10.000 rotações/minuto por 25 minutos e filtradas em filtros milipore $0,45 \mu \mathrm{m}$ de poro por $25 \mathrm{~mm}$. Os AGV foram quantificados usando-se cromatógrafo de fase gasosa Varian ${ }^{\circledR}$, modelo 2485 , com coluna de vidro de dois metros de comprimento e diâmetro de $1 / 8$ polegada com Chromosorb 101 (80 - 100 mesh).

As amostras de sangue foram obtidas de uma gota de sangue da ponta da cauda de cada animal, esta era colocada em uma fita descartável acoplada ao aparelho, o qual fazia a leitura da dosagem de glicose, na faixa de medição entre 10 e $600 \mathrm{mg} / \mathrm{dL}$. Para a dosagem de glicose foi utilizado o aparelho Advantage monitor de glicemia.

Dos bezerros machos, quatro de cada tratamento foram aleatoriamente selecionados e abatidos. Os animais foram pesados e em seguida foram eutanasiados por de eletrocussão precedida de anestesia geral. A avaliação anato-patológica do rúmen foi realizada após a exposição do tubo digestivo do animal. Foram feitas ligaduras duplas nas porções distais do esôfago e do abomaso. Em seguida foram feitas ligaduras duplas paralelas nas porções proximal e distal do reticulorumen, do omaso, e do abomaso, sendo os compartimentos separados, para posterior abertura e retirada dos resíduos alimentares, seguido de lavagem em água corrente. Em seguida, o rúmen de cada bezerro foi inspecionado para avaliação anato-patológica descritiva.

Amostras dos ingredientes e do concentrado foram coletadas para a determinação da MS, PB, EE, cinzas, Ca e P de acordo com a AOAC (1980); FDN e FDA foram analisadas de forma sequencial, pelo método de Van Soest et al. (1991), as análises foram executadas no laboratório de nutrição do Departamento de Zootecnia 


\begin{tabular}{|c|c|c|c|c|c|}
\hline \multirow{2}{*}{ Ingredientes } & & \multicolumn{4}{|c|}{ Concentrado } \\
\hline & & \multicolumn{2}{|r|}{ Milho (\%) } & \multicolumn{2}{|r|}{ Polpa cítrica (\%) } \\
\hline Milho & & \multicolumn{2}{|r|}{66} & \multicolumn{2}{|r|}{-} \\
\hline Polpa cítrica & & \multicolumn{2}{|r|}{-} & \multicolumn{2}{|r|}{64,2} \\
\hline Farelo de soja & & \multicolumn{2}{|r|}{25} & \multicolumn{2}{|r|}{34} \\
\hline Calcário & & \multicolumn{2}{|r|}{1,5} & \multicolumn{2}{|r|}{-} \\
\hline Fosfato bicálcico & & \multicolumn{2}{|r|}{0,5} & \multicolumn{2}{|r|}{0,8} \\
\hline Sal mineral & & \multicolumn{2}{|r|}{1} & \multicolumn{2}{|r|}{1} \\
\hline Sabugo de milho & & \multicolumn{2}{|r|}{6} & \multicolumn{2}{|r|}{-} \\
\hline Rumensin & & \multicolumn{2}{|r|}{0,05} & \multicolumn{2}{|r|}{0,05} \\
\hline \multicolumn{6}{|c|}{ Composição dos ingredientes } \\
\hline Item ( $\%$ da matéria seca) & Milho grão & Polpa cítrica & Farelo de soja & Concentrado milho & Concentrado polpa cítrica \\
\hline MS (\% matéria natural) & 88,25 & 90,14 & 89,00 & 88,68 & 89,90 \\
\hline Proteína bruta & 9,74 & 6,84 & 52,08 & 20,85 & 20,80 \\
\hline Extrato etéreo & 3,40 & 2,37 & 2,17 & - & - \\
\hline Extrativo não nitrogenado & 82,24 & 69,40 & 32,62 & - & - \\
\hline Cinzas & 1,43 & 6,66 & 6,62 & 4,53 & 6,78 \\
\hline Calcio & 0,26 & 1,26 & 0,30 & 0,99 & 1,42 \\
\hline Fósforo & 0,31 & 0,09 & 0,64 & 0,55 & 0,52 \\
\hline Fibra em detergente neutro & 13,50 & 25,99 & 13,10 & 25,39 & 22,42 \\
\hline Fibra em detergente ácido & 4,55 & 26,05 & 9,23 & 4,97 & 15,27 \\
\hline
\end{tabular}

da Escola de Veterinária da Universidade Federal de Minas Gerais.

Para nenhuma das variáveis analisadas foram observadas diferenças entre os sexos, sendo os dados agrupados por tratamento. Os parâmetros de consumo e desempenho dos animais que não foram submetidos às coletas de líquido ruminal e de sangue foram avaliados semanalmente. Os parâmetros de líquido ruminal e de sangue foram avaliados por período de coleta. Os dados coletados nos períodos (semana ou dia de coleta) foram analisados por medidas repetidas no tempo pelo PROC MIXED. Tratamento, período, tratamento $\mathrm{x}$ período, foram considerados como efeitos fixos e o animal dentro de tratamento foi considerado como efeito aleatório. Diferenças entre tratamentos e o PCN foram analisados pelo PROC GLM (SAS, versão 9.0, SAS Institute Inc. Cary, NC), por comparação de média pelo teste de Tukey. O PCN foi considerado como covariável. Para as avaliações anato-patológicas dos compartimentos do rúmen foi adotada análise estatística descritiva. As diferenças entre as médias foram consideradas significativas para $\mathrm{p} \leq 0,05$.

\section{RESULTADOS E DISCUSSÃO}

Observa-se que o consumo de concentrado foi pequeno no primeiro mês de vida (média de $127 \mathrm{~g}$ MS/ dia), aumentando rapidamente a partir da quinta semana de idade em ambos os tratamentos (tabela II), podendo ser explicado pelo desaleitamento abrupto aos 30 dias de idade, deixando o concentrado como única fonte de alimento para os bezerros.
A interação observada entre os tratamentos e as semanas para o consumo de concentrado (g MS/d) demonstrou que a partir da quinta semana de idade ocorreu maior consumo pelo grupo que recebeu milho no concentrado em relação a aqueles que receberam polpa cítrica ( $\mathrm{p}=0,01$; tabela II). Menor aceitabilidade para rações com polpa cítrica foram relatadas por Van Horn et al. (1975) e Wing (1982) como motivo para a não inclusão deste alimento nas dietas de bezerros jovens. Porém, no presente estudo, até a quarta semana de idade os animais apresentaram consumos semelhantes, indicando que a aceitabilidade talvez não tenha sido a causa dessa diferença de consumo observada após a quinta semana de idade dos animais. Schalch et al. (2001) também não observaram problemas de aceitabilidade, porém, segundo os autores, a adição de $5 \%$ de leite em pó desnatado no concentrado pode ter aumentado a aceitabilidade da dieta pelos bezerros, uma vez que não foram observadas diferenças para o consumo de MS do concentrado entre as diferentes inclusões de polpa cítrica no concentrado ( 0 a $45 \%$ em substituição ao milho).

A concentração de glicose sanguínea foi semelhante entre os tratamentos (tabela II). Porém, foi observado que no primeiro mês de vida (coletas entre 10 e 30 dias de idade), bezerros de ambos os tratamentos apresentaram maiores concentrações de glicose $(82 \pm 33,36 \pm$ 12 e $36 \pm 13 \mathrm{mg} / \mathrm{dL}$, respectivamente a 14,24 e 34 dias de idade) quando comparado as coletas com 44 e 54 dias de idade, as quais apresentaram valores de $34 \pm$ 4 e $34 \pm 17 \mathrm{mg} / \mathrm{dL}$. Esses resultados demonstram que no primeiro mês de idade os animais estavam obtendo 
a energia necessária através da absorção e utilização da lactose fornecida pelo leite. Após o desaleitamento, as concentrações de glicose são reduzidas devido à mudança do substrato utilizado para gliconeogênese (lactose para propionato). Os valores encontrados são baixos e refletem a pequena capacidade de ingestão de alimentos sólidos durante a fase de aleitamento, indicando que o desaleitamento aos 30 dias de idade é um grande desafio para os animais.

O ganho de peso $(\mathrm{g} / \mathrm{d})$ foi semelhante entre os tratamentos (tabela II). Ao se observar a maior ingestão de concentrado para o grupo com milho a partir da quinta semana de idade e a similaridade de ganho de peso entre os tratamentos, o fornecimento de polpa cítrica proporcionou maior eficiência de aproveitamento pelos animais após o desaleitamento quando comparado ao fornecimento de concentrado a base de milho como fonte energética. Porém, é importante chamar atenção que no concentrado do grupo com milho houve inclusão de $6 \%$ de sabugo de milho para aproximar os teores de FDN e FDA entre as dietas (tabela I), além das diferenças na disponibilidade da proteína e do perfil de aminoácidos dos concentrados causados pela diferença na quantidade total de farelo de soja incluída, 9\% a mais no concentrado com polpa cítrica, os quais podem ser possíveis explicações para a similaridade entre os ganhos de pesos observados. Foi observado efeito quadrático do ganho de peso de

Tabela II. Consumo de concentrado (g MS/dia) e ganho de peso (g/dia) de bezerros ( $\mathrm{n}=14$ por tratamento) alimentados com concentrado à base de milho ou polpa cítrica até 59 dias de idade (Starter intake (g DM /day) and weight gain ( $g / d a y)$ calves ( $n=14$ per treatment) fed with starter based on corn or citrus pulp up to 59 days of age).

\begin{tabular}{|c|c|c|c|c|c|c|c|}
\hline \multirow{2}{*}{ Item } & & \multicolumn{2}{|c|}{ Tratamentos } & \multirow{2}{*}{ EPM } & \multicolumn{3}{|c|}{ Valor de $p$} \\
\hline & & Milho & Polpa cítrica & & $T$ & $S$ & $T \times S$ \\
\hline \multicolumn{2}{|c|}{ Consumo de concentrado (g MS/d) } & 593 & 411 & 29,9 & 0,01 & 0,01 & 0,01 \\
\hline \multicolumn{2}{|c|}{ Glicose sanguínea (mg/dL) } & 46,8 & 41,4 & 4,4 & 0,45 & 0,01 & 0,72 \\
\hline \multicolumn{2}{|c|}{ Ganho de peso (g/d) } & 186 & 136 & 15,5 & 0,20 & 0,01 & 0,14 \\
\hline \multirow{2}{*}{ Semana } & \multicolumn{2}{|c|}{ Tratamentos } & \multirow{2}{*}{\multicolumn{2}{|c|}{ EPM }} & \multirow{2}{*}{\multicolumn{3}{|c|}{ Valor de $p$}} \\
\hline & Milho & Polpa cítrica & & & & & \\
\hline \multicolumn{8}{|c|}{ Consumo de concentrado (g MS/dia) } \\
\hline 1 & 57 & 57 & & 9,6 & & 0,9 & \\
\hline 2 & 68 & 65 & & 7,1 & & 0,8 & \\
\hline 3 & 149 & 141 & & 11,8 & & 0,7 & \\
\hline 4 & 266 & 212 & & 28,9 & & 0,3 & \\
\hline 5 & 443 & 282 & & 36,1 & & 0,0 & \\
\hline 6 & 745 & 504 & & 54,0 & & 0,0 & \\
\hline 7 & 985 & 697 & & 57,8 & & 0,0 & \\
\hline 8 & 1222 & 793 & & 68,1 & & 0,0 & \\
\hline 9 & 1432 & 972 & & 66,6 & & 0,0 & \\
\hline$S$ & \multicolumn{7}{|c|}{ Ganho de peso (g/d) } \\
\hline 1 & \multicolumn{7}{|c|}{40,8} \\
\hline 2 & \multicolumn{7}{|c|}{221,3} \\
\hline 3 & \multicolumn{7}{|c|}{0,30} \\
\hline 4 & \multicolumn{7}{|c|}{0,42} \\
\hline 5 & \multicolumn{7}{|c|}{0,38} \\
\hline 6 & \multicolumn{7}{|c|}{212,7} \\
\hline 7 & \multicolumn{7}{|c|}{201,0} \\
\hline 8 & \multicolumn{7}{|c|}{329,8} \\
\hline 9 & \multicolumn{7}{|c|}{442,2} \\
\hline Efeito & \multicolumn{7}{|c|}{ Quadrático $(p>0,05)$} \\
\hline Período & \multicolumn{7}{|c|}{ Glicose sanguínea (mg/dL) } \\
\hline 14 dias & \multicolumn{7}{|c|}{81,8} \\
\hline 24 dias & \multicolumn{7}{|c|}{36,1} \\
\hline 34 dias & \multicolumn{7}{|c|}{36,6} \\
\hline 44 dias & & & & & & & \\
\hline 54 dias & & & & & & & \\
\hline Efeito qua & $>0,05)$ & & & & & & \\
\hline$S=$ Semar & tamientos; EPM & padrão da méc & & & & & \\
\hline
\end{tabular}


acordo com o aumento da idade dos animais, sendo observado menores valores de ganhos nas semanas 3, 4 e 5, período em que os animais apresentaram diarreia, em ambos os tratamentos.

Não foram observados efeitos dos tratamentos sobre o $\mathrm{pH}$ ruminal (tabela III), sendo verificados valores próximos ao relatado por Schalch et al. (2001), os quais verificaram média de 6,1 para bezerros que receberam concentrado com $100 \%$ de substituição de milho pela polpa cítrica. A polpa cítrica possui maior potencial de produção de lactato e uma fermentação mais rápida, o que, consequentemente, gera menor $\mathrm{pH}$ ruminal quando comparado a dietas com farelo e grão de milho (Leiva et al., 2000), fato que não foi observado no presente estudo. Não foram observados efeitos dos tratamentos sobre as concentrações molares (mmol/L) de acetato, propionato e butirato, bem como para as concentrações de AGV total (tabela III). No entanto, observou-se que o grupo alimentado com milho apresentou maior percentual de propionato $(p=0,01)$ que o grupo alimentado com polpa cítrica. Já para o butirato, percentualmente a sua concentração foi maior $(\mathrm{p}<0,05)$ para o grupo alimentado com polpa cítrica no concentrado em relação aos animais que receberam milho no concentrado.

Os animais do tratamento com polpa cítrica apresentaram coloração mais escura do rúmen quando comparados aos animais que receberam milho no concentrado. O mesmo foi relatado por Schalch et al. (2001), os quais foram observadas ocorrências de escu- recimento das papilas e da mucosa da parede ruminal, de acordo com o aumento da quantidade de polpa cítrica na ração ( 0 a $45 \%$ em substituição ao milho), sem interferência nos resultados de consumo, desempenho, tamanho das papilas ruminais e desenvolvimento dos pré-estômagos dos bezerros.

Áreas de aglutinação de papilas foram observadas no rúmen dos bezerros alimentados com milho no concentrado, diferente ao observado por Schalch et al. (2001). Os bezerros alimentados com polpa cítrica no concentrado apresentaram estrutura cilíndrica de cor preta em todos os compartimentos do pré-estomago, com aspecto duro, localizado entre as papilas, o qual formava estruturas compactadas de tamanho variável, atingindo até três $\mathrm{cm}$ de diâmetro. Devido à ocorrência somente nos bezerros que receberam polpa cítrica, suspeita-se que o aparecimento dessas estruturas seja decorrente da presença desse coproduto na dieta.

\section{CONCLUSÃO}

A utilização de polpa cítrica em substituição ao milho no concentrado de bezerros até 60 dias de idade alterou o consumo de concentrado, porém não reduziu desempenho dos animais e nem alterou os parâmetros da fermentação ruminal e de glicose plasmática. Animais do tratamento com polpa cítrica apresentaram coloração mais escura do rúmen, bem como uma estrutura cilíndrica de cor preta em todos os compartimentos do pré-estomago. Estudos complementares de digestibilidade das dietas e análises de custos são necessários

Tabela III. Médias de pH e das concentrações molares e percentuais de acetato, propionato, butirato do fluido ruminal, de bezerros ( $n=4$ por tratamento) desaleitados aos 30 dias de idade, alimentados com concentrado a base de milho ou polpa cítrica até 59 dias de idade (Average $\mathrm{pH}$ and molar and percentagens concentrations of acetate, propionate, and butyrate in rumen fluid of calves ( $n=4$ per treatment) weaned at 30 days of age, fed with starter based on corn or citrus pulp up to 59 days of age).

\begin{tabular}{|c|c|c|c|c|c|c|c|}
\hline \multirow{2}{*}{ Item } & & \multicolumn{2}{|c|}{ Tratamentos } & \multirow{2}{*}{ EPM } & \multicolumn{3}{|c|}{ Valor de $p$} \\
\hline & & Milho & Polpa citrica & & $\mathrm{T}$ & $\mathrm{P}$ & $\mathrm{T} \times \mathrm{P}$ \\
\hline $\mathrm{pH}$ & & 5,9 & 5,8 & 0,08 & 0,74 & 0,09 & 0,66 \\
\hline \multicolumn{8}{|l|}{ AGV (mmol/L) } \\
\hline Acetato & & 3,4 & 4,2 & 0,34 & 0,32 & 0,01 & 0,29 \\
\hline Propionato & & 3,5 & 1,8 & 0,34 & 0,12 & 0,01 & 0,46 \\
\hline Butirato & & 0,6 & 0,8 & 0,09 & 0,57 & 0,01 & 0,11 \\
\hline Total $(\mathrm{mmol} / \mathrm{L})$ & & 7,6 & 6,8 & 0,69 & 0,83 & 0,68 & 0,83 \\
\hline \multicolumn{8}{|l|}{ AGV (\%) } \\
\hline Acetato & & 47,8 & 59,7 & 2,33 & 0,06 & 0,99 & 0,43 \\
\hline Propionato & & 44,9 & 25,8 & 2,09 & 0,01 & 0,16 & 0,55 \\
\hline Butirato & & 7,3 & 9,8 & 0,79 & 0,04 & 0,03 & 0,34 \\
\hline \multirow{2}{*}{ Período (P) } & \multicolumn{3}{|c|}{ AGV (mmol/L) } & \multicolumn{4}{|c|}{ AGV (\%) } \\
\hline & Acetato & Propionato & Butirato & \multicolumn{4}{|c|}{ Butirato } \\
\hline 14 dias & 1,3 & 1,0 & 0,2 & \multicolumn{4}{|c|}{4,1} \\
\hline 24 dias & 3,2 & 2,3 & 0,5 & \multicolumn{4}{|c|}{7,2} \\
\hline 34 dias & 3,6 & 2,4 & 0,6 & \multicolumn{4}{|c|}{9,8} \\
\hline 44 dias & 5,0 & 4,1 & 0,8 & \multicolumn{4}{|c|}{8,7} \\
\hline 54 dias & 5,7 & 4,1 & 1,2 & \multicolumn{4}{|c|}{11,7} \\
\hline Efeito & Linear $(p>0,05)$ & Linear $(p>0,05)$ & Linear $(p>0,05)$ & \multicolumn{4}{|c|}{ Linear $(p>0,05)$} \\
\hline
\end{tabular}

$\mathrm{P}=$ Periodo; $\mathrm{T}=$ Tratamientos; $E P M=$ Erro padrão da média; $\mathrm{AGV}=$ Ácidos graxos voláteis. 
para correta indicação desse coproduto na dieta de bezerros leiteiros durante a fase de aleitamento.

\section{BIBLIOGRAFIA}

AOAC. Association of Official Analytical Chemists. 1980. Official methods of analisys. $16^{\text {th }} \mathrm{ed}$. Washington, D.C.

Azevedo, R.A.; Rufino, L.M.D.; Santos, A.C.R.; Silva, L.P.; Bonfá, H.C.; Duarte, E.R. e Geraseev, L.C. 2012. Desempenho de cordeiros alimentados com inclusão de torta de macaúba na dieta. Pesq Agropec Bras, 47: 1663-1668.

Azevedo, R.A.; Santos, A.C.R.; Ribeiro Júnior, C.S.;Santos, F.P.C.; Araújo, L.; Bicalho, F.L.; Fonseca, L.M. e Geraseev, L.C. 2014. Desempenho de vacas em lactação alimentadas com dietas contendo torta de macaúba. Arq Bras Med Vet Zootec, 66: 211-218.

Costa, F. M.J.; Dias Júnior, G.S.; Zacaroni, O.F.; Santos, J.F.; Pereira, R.A.N. e Pereira, M.N. 2014. Silagem de grãos úmidos de milho de textura dura ou macia em dietas com polpa cítrica para vacas em lactação. Arq Bras Med Vet Zootec, 66: 203-210.

Harris Jr., B. and Staples, C.R. 1989. Feeding by products feedstuffs to dairy cattle. Florida Cooperative Extension Service/IFAS/University of Florida, DS.

Javed, M.Z.; Sharif, M.; Bhatti, S.A.; Bilal, M.Q.; Ahmed, F., Ahmad, F.; Saif-ur-Rehman, M. and Tariq, M. 2016. Nutrient intake, nitrogen balance and growth performance in buffalo calves fed citrus pulp as a concentrate source. African J Agric Res, 11: 2562-2568.

Leiva, E.; Hall, M.B. and Van Horn, H.H. 2000. Performance of dairy cattle fed citrus pulp or corn products as sources of neutral detergentsoluble carbohydrates. J Dairy Sci, 83: 2866-2875.

Mendes Neto, J.; Campos, J.M.S. e Valadares Filho, S.C. 2007. Comportamento ingestivo de novilhas leiteiras alimentadas com polpa cítrica em substituição ao feno de capim-tifton 85. Rev Bras Zootecn, 36: 618-625.

Nussio, C.M.B.; Santos, F.A.P.; Pires. A.V. Simas, J.M.C. e Zopollato, M. 2002. Fontes de amido de diferentes degradabilidades e sua substituição por polpa de citrus em dietas para vacas leiteiras. Acta Sci, 24: 1079-1086.
Peixoto, E.L.T.; Morenz, M.J.F.; Fonseca, C.E.M.; Moura, E.S.; Lima, K.R.; Lopes, F.C.F. and Cabral, L.S. 2015. Citrus pulp in lamb diets: intake, digestibility, and ruminal parameters. Semina: Ciênc Agrár, 36: 3421-3430.

Pimentel, P.G.; Reis, R.B.; Leite, L.A.; Campos, W.E.; Neiva, J.N.; Saturnino, H.M. e Coelho, S.G. 2012. Parâmetros da fermentação ruminal e concentração de derivados de purina de vacas em lactação alimentadas com castanha de caju. Arq Bras Med Vet Zootec, 64: 959-966.

Pires, A.J.V.; Vieira, V.F.; Silva, F.F.; Veloso, C.M.; Souza, A.F.; Oliveira, T.N; Santos, C.L. e Carvalho, G.G.P. 2005. Níveis de farelo de cacau (Theobroma cacao) na alimentação de bovinos. REDVET, 6: 1-10.

Santos, F.A.P.; Menezes Júnior, M.P.; Simas, J.M.C.; Pires, A.V. e Nussio, C.M.B. 2001. Processamento do grão de milho e sua substituição parcial por polpa de citros peletizada sobre o desempenho, digestibilidade de nutrientes e parâmetros sanguíneos. Acta Sci, 23: 923-931.

Santos, G.T.; Lima, L.S.; Schogor, A.L.B.; Romero, J.V.; De Marchi, F.E.; Grande, P.A.; Santos, F.S. and Kazama, R. 2014. Citrus pulp as a dietary source of antioxidants for lactating Holstein cows fed highly polyunsaturated fatty acid diets. Asian-Australasian J Anim Sci, 27: 1104.

Schalch, F.J.; Schalch, E.; Zanetti, M.A. e Brisola, M.L. 2001. Substituição do milho em grão moído pela polpa cítrica na desmama precoce de bezerros leiteiros. Rev Bras Zootecn, 30: 80-285.

Silva, H.G.O.; Pires, A.J.V.; Silva, F.F. Veloso, C.M.; Carvalho, G.G.P.; Cezário, A.S. e Santos, C.C. 2005. Farelo de cacau (Theobroma cocoa L.) e torta de dendê (Elaeis guineensis, Jacq) na alimentação de cabras em lactação: consumo e produção de leite. Rev Bras Zootecn, 34: 1790-1798.

Van Soest, P.J.; Robertson, J.B. and Lewis, B.A. 1991. Methods for dietary fiber, neutral detergent fiber and nonstarch polysaccharides in relation to animal nutrition. J Dairy Sci, 10: 3583-3597.

Wing, J.M. (Ed.). 1982. Citrus feedstuffs for dairy cattle. University of Florida. Gainesville. (Bulletin 829). 